\title{
Fostering population health research in NSW: the role of research infrastructure
}

\author{
Sarah V. Thackway ${ }^{\mathrm{A}}$ and Jo Mitchell ${ }^{\mathrm{A}, \mathrm{B}}$ \\ ${ }^{\mathrm{A}}$ Centre for Epidemiology and Research, \\ NSW Department of Health \\ ${ }^{\mathrm{B} C o r r e s p o n d i n g ~ a u t h o r . E m a i l: ~ j m i t c @ d o h . h e a l t h . n s w . g o v . a u ~}$
}

Research infrastructure - the assets, facilities and services that support research and maintain the capacity of researchers to undertake research - is an important contributor to research excellence. ${ }^{1}$ A key theme in the 2008 review of public health research funding in Australia was the need for strategic investment in public health research infrastructure, including centres of research excellence, large scale assets such as cohort studies, disease registers, data linkage and survey facilities, and the career development of researchers. ${ }^{2}$

Several infrastructure funding programs at the federal and state government level provide research organisations with resources to help meet indirect costs that are not met by research grants. There is also a move towards more strategic investment in research infrastructure, for example the National Collaborative Research Infrastructure Strategy. ${ }^{1}$ Such investments, which are often collaborative, are designed to create research assets, promote data access for a wide range of researchers and avoid duplication of effort.

NSW Health provides support for population health research infrastructure. ${ }^{3}$ Strategies include funding research organisations relevant to NSW population health priorities, population health surveys and statewide data collections, research capacity building programs and supporting other research assets (Table 1).

The infrastructure supported by NSW Health promotes the generation and use of population health research in several ways. For example:

- Research centres build the evidence base around New South Wales (NSW) priority areas and facilitate the adoption of research findings in policy and programs through the synthesis and dissemination of research findings and provision of advice in strategy development.

- The ongoing monitoring of population health and the establishment of research asset studies provide information about trends in health, health behaviours and attitudes. ${ }^{4}$ Surveillance data and linked data sets can be made available to researchers, allowing costeffective analyses on large population samples. The outputs of these analyses help to test hypotheses, identify population health issues and inform the evaluation of policies and programs.

- Capacity building strategies increase workforce skills in commissioning and undertaking research and in using research evidence in policy and practice. ${ }^{3}$

The following three case studies illustrate how infrastructure fosters better population health research in NSW. The first describes how the Physical Activity, Nutrition and Obesity Research Group, a NSW Health funded research centre, has increased the generation and use of policyrelevant research. The second and third case studies explain how the 45 and Up Study and the Centre for Health Record Linkage encourage large scale, efficient and timely research. All three initiatives include a focus on developing links and partnerships between policy makers and researchers.

Research infrastructure contributes to the generation and use of high quality, policy-relevant research, leading to improved policy and program effectiveness, better population health and reduced health inequity. While NSW Health makes a significant investment in this area, increasing the impact of this investment (e.g. through fostering greater awareness of major research assets and how to use them and determining the best investment mix) remains an ongoing challenge. Increasing the impact of this investment is a major focus of work within NSW Health over the next 5 years. ${ }^{3}$

\section{References}

1. Department of Innovation. Industry and Science. Strategic Roadmap for Australian Research Infrastructure. Canberra: Australian Government; 2008.

2. National Health and Medical Research Council. Report of the Review of Public Health Research Funding in Australia, December 2008. Canberra: Commonwealth of Australia; 2009.

3. Centre for Epidemiology and Research. Promoting the generation and effective use of population health research in NSW: a Strategy for NSW Health 2011-2015. North Sydney: NSW Department of Health; 2010.

4. NSW Health. New South Wales School Students Health Behaviours Survey. Available from: http://www.health.nsw.gov.au/ publichealth/surveys/hss/index.asp (Cited 30 December 2010.) 


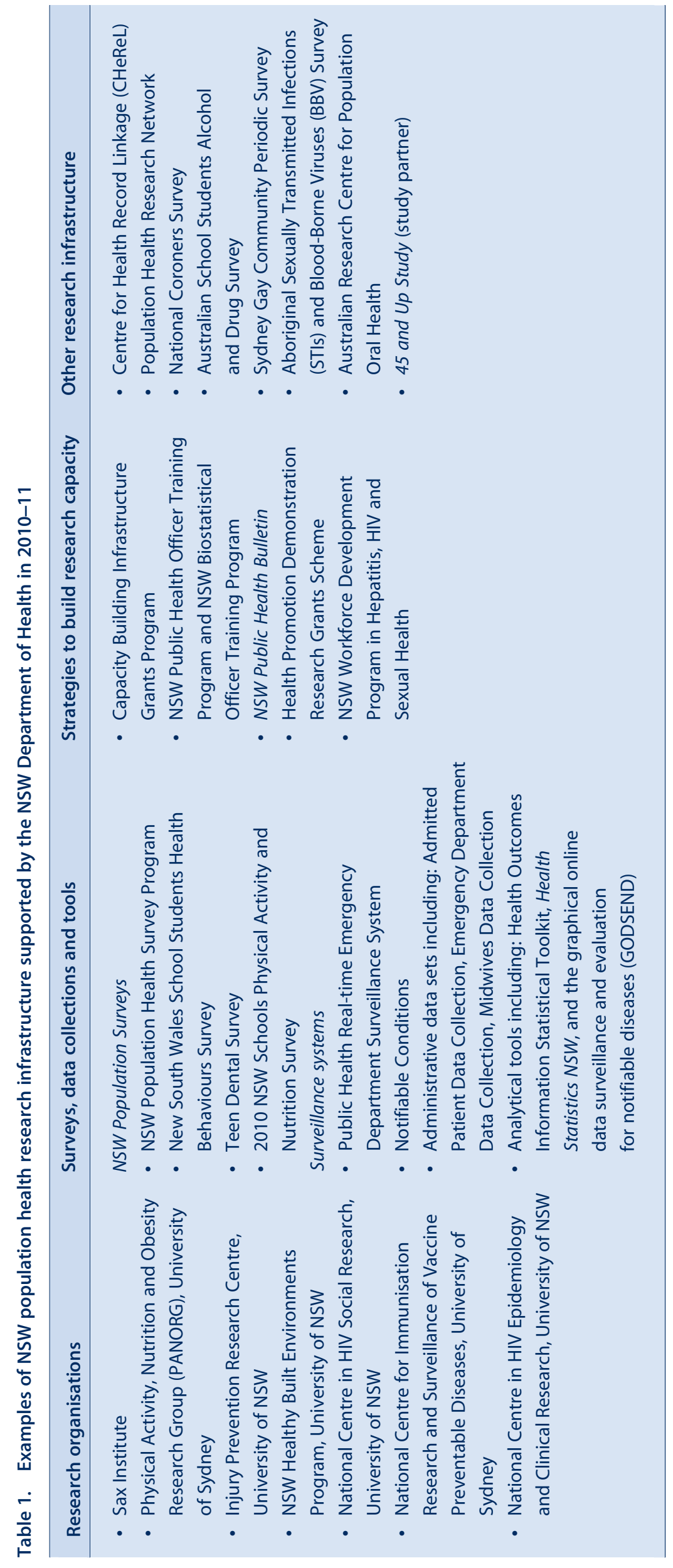

